\title{
Assistente de Cena de Crime: Solução Para Auxílio à Investigação de Homicídios
}

\author{
Mateus Antonio Amorim ${ }^{1}$, João Venceslau ${ }^{1}$, Pedro Carvalho ${ }^{1}$, \\ Nélio Cacho ${ }^{2}$, Frederico Lopes ${ }^{1}$, Eiji Adachi ${ }^{1}$, José Alex Lima ${ }^{1}$ \\ ${ }^{1}$ Instituto Metrópole Digital \\ Universidade Federal do Rio Grande do Norte (UFRN) - Natal, RN - Brasil \\ ${ }^{2}$ Departamento de Informática e Matemática Aplicada \\ Universidade Federal do Rio Grande do Norte (UFRN) - Natal, RN - Brasil \\ mateuscfc8@outlook.com, jv.venceslau.c@gmail.com,
carvalho.pedro.cardoso@gmail.com, neliocacho@dimap.ufrn.br,
fred@imd.ufrn.br, eijiadachi@imd.ufrn.br, j.alex.medeiros@gmail.com
}

Resumo. Este artigo traz a apresentação de uma ferramenta feita com o propósito de auxiliar a coleta de informações em locais nos quais um homicídio pode ter acontecido. A ferramenta, denominada Assistente de Cena de Crime, foi desenvolvida com as funcionalidades de criação de ocorrências a partir de dados da API do Centro Integrado de Operações de Segurança Pública, preenchimento de formulários com informações que auxiliarão a investigação criminal, captura precisa de geolocalização do evento e emissão de documentos pertinentes a cada ocorrência. O sistema também trabalha sem conexão com a internet e em dispositivos móveis, sendo desenvolvido a partir dos frameworks React, baseado em JavaScript, e Spring, baseado em Java.

\begin{abstract}
This paper presents a tool made for the purpose of helping data collection on places where a homicide may have occurred. The tool, which is called Assistente de Cena de Crime, was developed with the functionalities of creating occurrences based on data from the Integrated Center of Public Security Operations API, filling of forms with information that will help criminal investigation, precise capture of geolocation and emission of documents from each occurrence. The system also works without internet connection and on mobile devices. It was made using React, a JavaScript-based framework, and Spring, a Java-based framework.
\end{abstract}

\section{Introdução}

Por negar completamente o direito à vida - um direito básico e fundamental em qualquer sociedade livre - e por evidenciar o potencial agressivo e destrutivo que qualquer indivíduo numa sociedade pode ter, crimes de homicídio costumam ter grande choque prejudicial na sociedade. De fato, crimes de homicídio são preocupantes não apenas pelas vidas perdidas, mas também pelo impacto negativo que têm na saúde e na percepção de segurança e bem estar de uma população [Souza et al. 2005].

Nos últimos anos, o número de vítimas por homicídio vem aumentando significativamente no Brasil. De acordo com dados do Atlas da Violência, produzido pelo Instituto de Pesquisa Econômica Aplicada em parceria com o Fórum Brasileiro de Segurança 
Pública (FBSP), no ano de 2006 a taxa de homicídios no Brasil era de 23,7 para cada 100 mil habitantes, e em 2016 essa taxa aumentou para aproximadamente 30,3 . ${ }^{1}$ Estes valores são aproximadamente 30 vezes a taxa da Europa. No estado do Rio Grande do Norte, no período entre 2006 e 2016, a taxa de homicídios passou de 14,9 a 53,4, o que representa uma variação de aproximadamente $+256,9 \%$, sendo esta a pior variação dentre todas as unidades federativas brasileiras.

No Brasil, a investigação de homicídios é responsabilidade da Polícia Civil e tem início na fase chamada de investigação preliminar, a qual compreende todos os procedimentos de investigação e de coleta de vestígios realizados no primeiro momento em que a polícia recebe a informação da ocorrência de um homicídio, até os trabalhos na cena do crime, imediatamente após a chegada dos primeiros agentes policiais ao local.

Apesar de sua importância para a investigação criminal, um estudo realizado por Mingardi e Figueiredo [Mingardi and Figueiredo 2005] em delegacias do estado de São Paulo aponta que o modelo investigativo, observado nestas delegacias, começa a apresentar problemas já em sua fase preliminar. Tal estudo aponta adversidades desde a demora das equipes policiais chegarem ao local do crime, passando pelo inadequado isolamento da área do crime, até a falta de equipamentos adequados para a realização do registro e armazenamento de informações coletadas na cena do crime. Sendo o último apontado pela equipe da Divisão Especializada em Homicídios da Polícia Civil do Rio Grande do Norte (DHPP) como um dos problemas enfrentados atualmente por seus delegados e investigadores durante a fase de investigação preliminar. Em seu modo de operação atual, os investigadores da DHPP dirigem-se ao local do crime munidos de pranchetas e formulários em papel para fazer despachos e coletar informações relevantes que subsidiarão o relatório confeccionado pelo delegado que preside a investigação.

Este artigo apresenta uma solução desenvolvida pelo projeto Smart Metropolis $^{2}$ da Universidade Federal do Rio Grande do Norte (UFRN), iniciativa que tem como objetivo a aplicação de conceitos de Cidades Inteligentes como forma de modernizar e facilitar situações do cotidiano urbano, em parceria com a DHPP. Tal solução provê um sistema de informação com forte integração com a plataforma de Cidades Inteligentes implantada em Natal e visa apoiar a coleta de dados em locais de crime (como cadastro de vitimas, evidencias, detalhes do ambiente, depoimento de testemunhas e outros dados essenciais), a geração automática de relatórios sobre a cena de crime(sendo necessária apenas descrições mais especificas de imagens coletadas) e de guias de solicitação de exames periciais, que são atividades essenciais realizadas pela equipe da DHPP. A informatização dos processos realizados durante a investigação preliminar visa dar-lhes maior celeridade, agilizando a investigação de homicídios como um todo. Esta aplicação suporta as atividades de investigação de homicídios através da informatização dos processos investigativos, bem como a integração com sistemas de outros órgãos públicos também envolvidos com esse tipo de crime.

Inicialmente esse artigo aborda conceitos sobre a investigação de homicidios (Seção 2). Posteriormente, na Seção 3, o artigo apresenta a ferramenta Assistente de Cena de Crime (ACC), desenvolvida no projeto Smart Metropolis. A Seção 4 apresenta

\footnotetext{
${ }^{1}$ http://ipea.gov.br/atlasviolencia/

${ }^{2} \mathrm{http}: / /$ smartmetropolis.imd.ufrn.br/
} 
os trabalhos relacionados e a Seção 5 apresenta as conclusões deste artigo.

\section{Investigação de Homicídios}

A investigação só ocorre em caso de morte violenta, ou seja, quando há homicídio, suicídio, acidente (afogamento, overdose de drogas, acidente de trabalho, acidente de carro etc.) ou quando há acontecimentos suspeitos, não sendo possível visualizar com clareza sua causa, necessitando então de uma investigação para maiores esclarecimentos. No que se refere aos crimes de homicídio, tipicamente divide-se a investigação criminal em duas etapas distintas e complementares: (1) Investigação Preliminar - conforme definido no Caderno Temático de Referência de Investigação Criminal de Homicídios [Moraes et al. 2014], investigação preliminar é todo procedimento de registro e análise de elementos objetivos e subjetivos, realizado na cena do crime, imediatamente após a chegada dos primeiros policiais ao local; e (2) Investigação de Seguimento - etapa que abarca os procedimentos investigativos e cartoriais realizados pela polícia desde o encerramento dos trabalhos preliminares até a conclusão do inquérito (resultando, preferencialmente, na completa elucidação do crime e de suas circunstâncias, com obtenção de autoria e materialidade).

Tipicamente, o trabalho realizado em local de crime envolve a atuação de três atores distintos: agentes da Polícia Militar, investigadores da Polícia Civil e peritos do Instituto Médico Legal. Para facilitar o seu entendimento, a investigação preliminar pode ser dividida nas fases apresentadas na Figura 1 e que são detalhadas a seguir.

Figura 1. Processo de Investigação de Homicídios

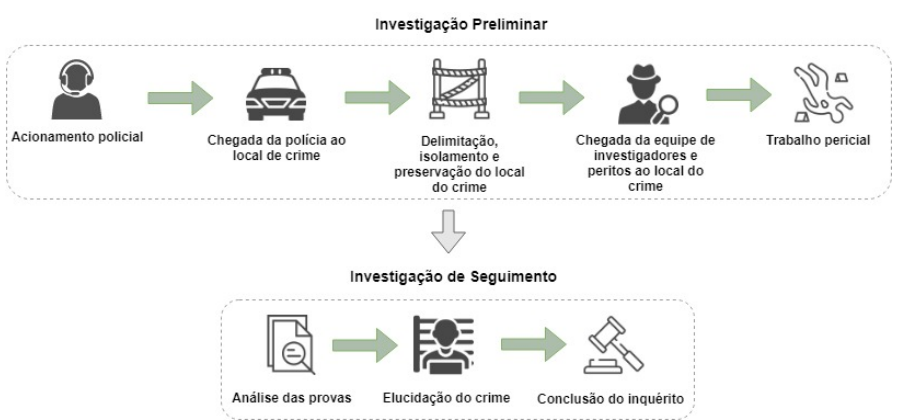

Acionamento policial e Chegada da polícia ao local do crime. A investigação de um homicídio tem início no momento em que a Polícia Militar recebe um chamado informando sobre a ocorrência do crime. $\mathrm{O}$ agente que recebe o chamado coleta informações sobre o fato criminoso (endereço, hora da ocorrência, identidade da vítima, paradeiro do autor, etc.) e sobre a pessoa que está fazendo o acionamento policial (identidade, endereço, relação com o fato, etc.). Estas informações são repassadas para os funcionários direcionados ao local do crime.

Chegando ao possível local do homicídio, os agentes da Polícia Militar avaliam as condições de segurança do ambiente na tentativa de detectar possíveis ameaças às pessoas presente e à própria equipe. Se for constatado que existem pessoas feridas ainda vivas, deve-se providenciar o atendimento médico. Quando possível, já neste momento, os agentes da Polícia Militar que estão prestando socorro tentam obter informações sobre a autoria, a motivação e a dinâmica do crime. 
Delimitação, isolamento e preservação do local do crime. Constatando-se a ocorrência de óbito no local do crime, os agentes da Polícia Militar devem proceder com a delimitação, o isolamento e a preservação do local. A ação consiste na avaliação, ainda que preliminar, da extensão do local dos fatos criminosos. Em seguida, devem isolar a área com barreiras físicas para impedir o trânsito de pessoas não autorizadas nos locais, bem como a alteração de qualquer objeto possivelmente relacionado ao crime.

Chegada da equipe de investigadores e peritos ao local do crime. Ao chegar a uma cena de crime, cabe aos investigadores da Polícia Civil acompanhar a realização dos trabalhos periciais, coletando o máximo de dados possíveis, bem como o levantamento de dados sobre o crime, como a localização e identificação de possíveis testemunhas ou suspeitos, por exemplo. Cabe ainda aos investigadores zelar pela idoneidade dos vestígios materiais presentes na cena do crime.

Trabalho pericial. O trabalho pericial é realizado por funcionários do Instituto Médico Legal. Na cena de crime, os elementos objetivos produzidos pelos peritos ajudam os investigadores a melhor compreender a dinâmica do homicídio e a identificar possíveis hipóteses a serem exploradas a partir dos vestígios materiais eventualmente coletados. Tipicamente, os elementos produzidos, geralmente, são: fotografias, croquis, impressões digitais, vestígios físicos, etc.

Por fim, o responsável pela investigação preliminar deve montar um relatório descrevendo os fatos e acontecimentos descobertos durante esta fase, o qual será utilizado como ponto de partida pelo encarregado pela investigação de seguimento (é importante ressaltar que, tipicamente, ambos os momentos não compartilham do mesmo delegado incubado para a tarefa). Desta forma, a investigação de seguimento tomará por base as informações colhidas no local de crime e deve ser concluída com definição da autoria dos fatos e comprovação do evento. Sem a etapa preliminar o estágio seguinte fica prejudicado, sendo muito difícil alcançar na totalidade seu objetivo, que é a condenação do autor na Justiça Criminal.

Todas as atividades realizadas pelos investigadores da Polícia Civil no local do crime e, posteriormente, nas delegacias, para produzir os relatórios e guias de exames periciais, ainda não contam com suporte de sistemas de software. Ocasionado um trabalho braçal e arcaico, sendo base dos relatos dos delegados da DHPP como problemática.

\section{Assistente de Cena de Crime \& Cidades Inteligentes}

O desenvolvimento de uma aplicação para assistência policial no ambiente de cidades inteligentes demandam requisitos e funcionalidades especificas que serão tratadas nas seções seguintes.

\subsection{Requisitos}

O desenvolvimento do ACC foi realizado com base nos principais requisitos de uma aplicação para cidades inteligentes: integração: pois em versões futuras busca-se integração com o DETRAN, busca de informações a partir das placas, CIOSP para geolocalização do crime, além do ITEP e do SisPol para outras informações, conectividade: pois é necessário que funcione em locais sem acesso a internet, escalabilidade: uma possível arquitetura em nuvem para permitir maior escalabilidade no futuro e usabi- 
lidade: pois dado o ambiente estressante onde os dados são coletados, é necessária uma usabilidade simples e direta, que não cause mais stress aos usuários.

\subsection{Arquitetura}

No sentido de atender aos requisitos descritos acima, a solução foi desenvolvida a partir de duas tecnologias distintas para o front-end e para o back-end: React, um framework de desenvolvimento web baseado em JavaScript e Spring, um framework de criação de sistemas baseado em Java. Para o armazenamento de dados, foi utilizado o PostgreSQL como banco de dados principal da aplicação e, em cada dispositivo, no front-end, uma instância do PouchDB é criada para armazenar alterações locais e permitir acesso offline.

O back-end foi desenvolvido seguindo o padrão RESTful ${ }^{3}$ de sistemas web. Portanto, a comunicação entre front-end e back-end é feita a partir de requisições HTTP nos modelos PUT/POST/UPDATE/DELETE. Já a comunicação do back-end com o banco de dados é feita através de JPA (Java Persistence API). Uma representação da arquitetura implementada pode ser visto na Figura 2.

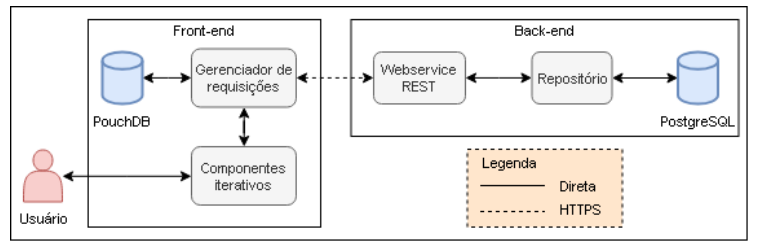

Figura 2. Arquitetura do sistema

\subsection{Principais responsabilidades do back-end:}

O back-end é responsável por realizar o armazenamento e processamento dos dados providos pelas demais aplicações do sistema, bem como por implementar as regras de negócio elicitadas, como as regras para geração do relatório de cena de crime e para a emissão das guias para exames periciais. Esta aplicação provê os seus serviços e integra-se aos demais componentes da arquitetura (sistemas externos e aplicações web e móvel) através de uma API REST. Os componentes internos desta aplicação são três:

- PostgreSQL: sendo este um object-relational database management system (ORDBMS) para fazer o gerenciamento dos dados;

- Data access object (DAO): que servirá como uma interface para manipular as informações do banco de dados;

- Webservice API: que será a API REST de acesso para integração.

Os dados são guardadas em um banco criptografado, que apenas os administradores do sistema têm acesso, todas as informações são enviadas via https, garantindo a sua segurança durante a transmissão na rede.

\subsection{Principais responsabilidades do front-end:}

O front-end do ACC permite que delegados e agentes da DHPP interajam com os serviços providos pela aplicação back-end. O front-end foi implementado por meio do uso da tecnologia Progressive Web App, termo que caracteriza e descreve aplicações que fazem uso

\footnotetext{
${ }^{3}$ https://www.infoq.com/br/articles/rest-introduction
} 
de tecnologias implementadas em navegadores atuais, como service workers e manifestos de aplicativo web [Tandel and Jamadar 2018]. Uma das principais vantagens do PWA é o fato da aplicação precisar ser implementada apenas em uma plataforma web, sem a necessidade de criar uma versão alternativa para dispositivos móveis. Isso acontece pois o manifesto de aplicativo web define diretrizes para que o sistema, além de funcionar com a aparência de um app propriamente nativo, permitir com que o mesmo seja salvo no dispositivo a partir do acesso à URL. Os usuários têm uma visão administrativa dos dados coletados durante a fase de investigação preliminar, podendo visualizar, editar ou inserir novos dados, além de gerar o relatório de cena do crime, bem como as guias de exames periciais. $\mathrm{O}$ front-end também provê uma interface pela qual os usuários podem registrar dados ainda no local de crime.É utilizada a geolocalização via Open Street Maps, via o navegador onde a aplicação está rodando, ou o GPS do dispositivo móvel. Tal aplicação possibilita o uso de suas funcionalidades em modo offline, uma vez que alguns locais de crimes não possuem cobertura de rede com acesso a Internet. Desta forma, as informações são registradas localmente no dispositivo e sincronizadas posteriormente com a aplicação back-end.

\subsection{Fluxo da comunicação do Front-end com o Back-end}

A Figura 3 mostra um diagrama de atividades, que explica o fluxo no sistema desenvolvido, desde o login do usuário até a emissão de algum documento específico referente a uma ocorrência.

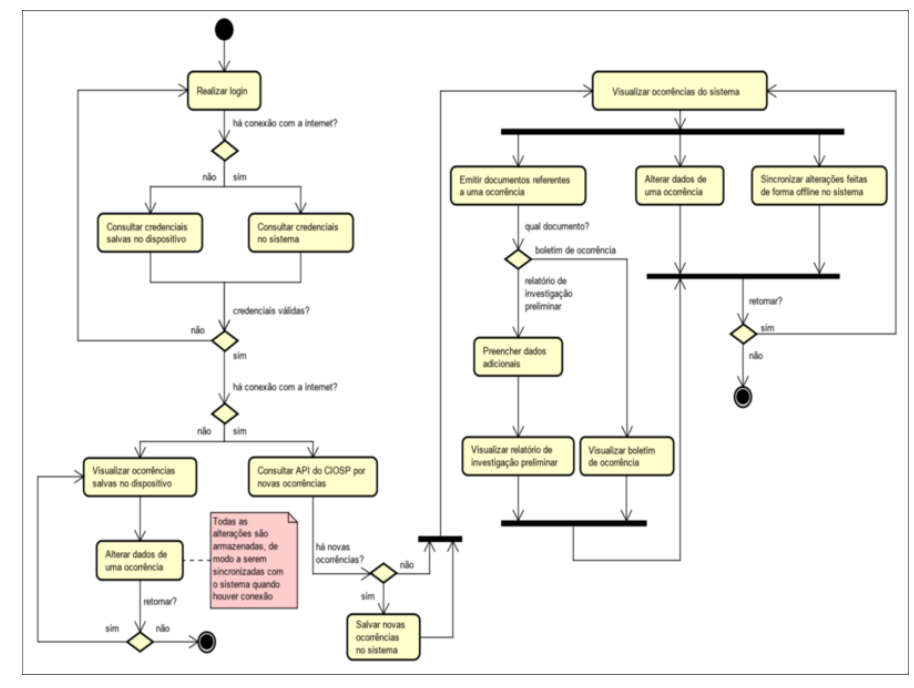

Figura 3. Diagrama de atividades do sistema

O fluxo é dependente do estado de conexão com a internet do usuário em dois principais pontos: no de login, na qual o usuário só poderá ter acesso offline caso já tenha acessado o sistema de maneira online pelo menos uma vez, e de funcionalidades, uma vez que o usuário só terá acesso a todas elas se estiver online. Em caso de perda de conexão foi utilizado um banco local, que guarda os dados modificados, e quando a conexão é reestabelecida, realiza as requisições para atualizar esses dados modificados.

Já a Figura 4 mostra um diagrama de casos de uso, enfatizando as funcionalidades disponíveis ao usuário e o diferenciando baseado no seu estado de conexão com a internet. Nota-se que, apesar de ser permitido ao usuário offline alterar dados das ocorrências, a 
sincronia dessas alterações só poderá ser feita quando houver conexão, ou seja, a partir do usuário online.

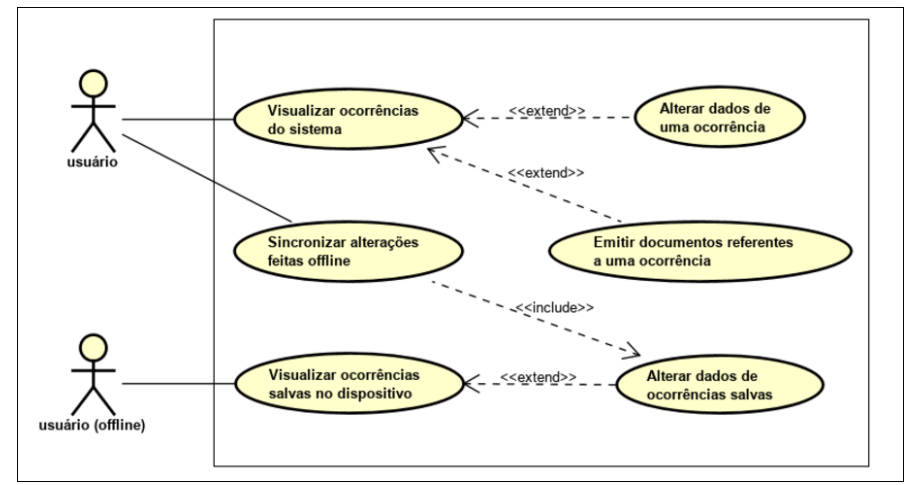

Figura 4. Diagrama de casos de uso do sistema

Ainda não há distinção entre papéis de usuários (e, consequentemente, permissões) em funcionalidades do sistema, portanto, todos os logins registrados terão acesso às funcionalidades descritas no diagrama.

\subsection{Homologação em Ambiente de Teste}

Vale salientar que todas as telas e funcionalidades que serão mencionadas foram validadas pelos delegados da DHPP. Além disso eles forneceram os formulários atualmente utilizados por eles. Durante a validação, tais delegados ainda propuseram algumas mudanças na organização dos formulários implementados, além de pequenas funcionalidades que gostariam de adicionar.

Durante o desenvolvimento da solução utilizamos dois ambientes. Em um deles se encontra a versão 'devel', que se remete à versão onde ocorrem as mudanças mais rapidamente e por isso mais sujeita a instabilidades, sejam por questão de bugs ou pela adição de novas funcionalidades. Tal ambiente é usado apenas pelos desenvolvedores. $\mathrm{O}$ outro ambiente hospeda a versão em homologação, versão mais estável utilizada pelos delegados da DHPP.

\subsubsection{Login}

É a primeira tela vista ao acessar a aplicação, independente do usuário. Essa tela possui os campos de inserção de matrícula e senha, além de um botão que redireciona para a tela de cadastramento de novo usuário. Ambos os campos possuem verificação de nulidade. Caso essa tela esteja sendo acessada de um dispositivo móvel, também haverá um botão com a opção de salvar a aplicação no dispositivo.

\subsubsection{Ocorrências}




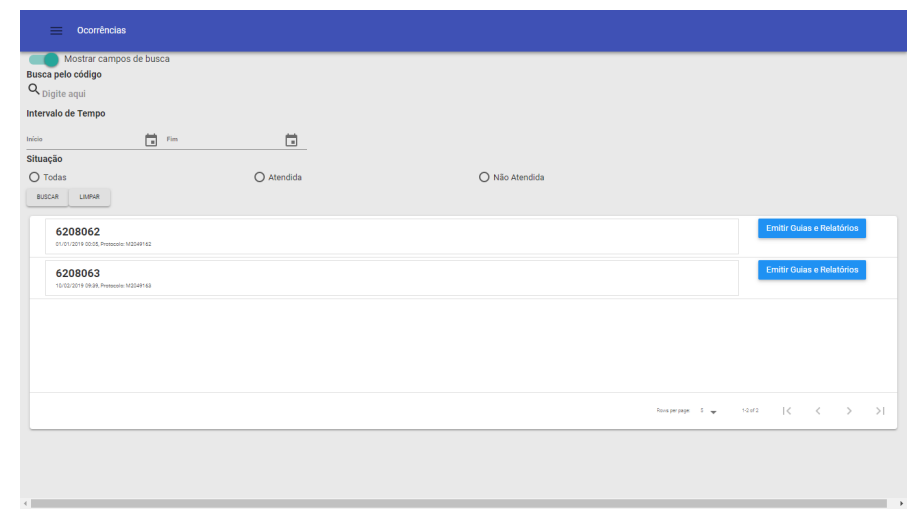

Figura 5. Tela de Ocorrências

Após o login ser efetuado, o usuário é direcionado para essa tela, onde é exibida a lista de ocorrências. Cada uma delas está atrelada a um botão, que leva para a tela responsável pela emissão de guias e relatórios da ocorrência correspondente. É possível habilitar os filtros para essa lista de ocorrências, podendo pesquisar ocorrências pelo seus códigos, intervalo de tempo em que ocorreram e sua situação (se foi atendida ou não). Ao solicitar os dados das ocorrências também é feito o armazenamento local dos dados para futuros acessos offline.

\subsubsection{Formulário}

Ao clicar em uma das ocorrências, o usuário será levado para essa tela. Aqui, ele poderá informar diversos aspectos do que aconteceu no local de crime, como vítimas, suspeitos, apreensões, entre outros, assim como ele o faria caso estivesse com o auto em mãos. A navegação entre seções é feita a partir da barra ao topo da tela. Algumas funcionalidades extras foram adicionadas, em relação ao documento-base, como o sistema de geolocalização.

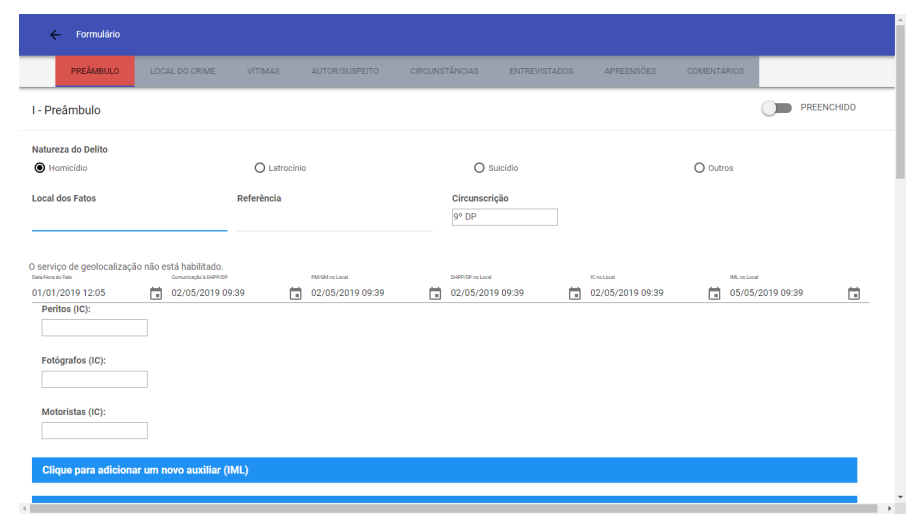

Figura 6. Tela inicial do Formulário

Cada aba trabalha de forma independente, assim é possível ter múltiplos usuários com a mesma ocorrência aberta e editarem abas diferentes ao mesmo tempo, o único cuidado que deve ser tomado é de não editarem a mesma aba pois apenas um deles terá os dados armazenados. Ao sair de uma aba os dados são enviados ao back-end para serem 
alterados no banco de dados, caso esteja offline, as mudanças são armazenadas localmente e posteriormente sincronizadas.

\subsubsection{Emissão de Relatórios}

Ao voltar para a pagina de listagem de ocorrências e selecionar a emissão de um relatório, são disponibilizados links para os diversos relatórios e guias que podem ser gerados a partir das informações da ocorrência, por exemplo o de Investigação Preliminar, serão carregados os dados atuais da ocorrência e será feita uma síntese para o preenchimento do relatório e apos o usuário preencher os dados restantes, será enviado para o back-end e ele irá gerar uma pagina com os dados da ocorrência e então retorna-la para o front-end.

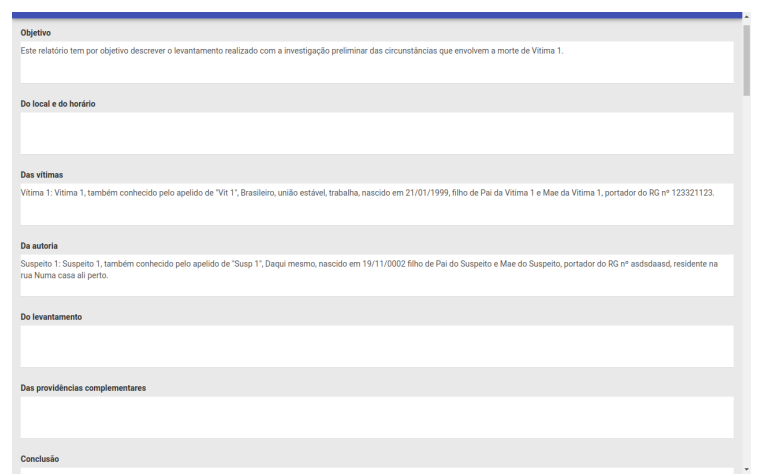

Figura 7. Tela para preenchimento adicional do RIP

\section{Trabalhos Relacionados}

Alguns aplicativos relacionados a segurança pública, mas que não dão suporte a atividades de investigação criminal são descritos pela literatura. A plataforma ROTA tem como principal objetivo a melhoria da segurança pública, tendo como funcionalidades o recebimento de ocorrências, gerenciamento e rastreamento das viaturas [Coelho et al. 2016]. O 'APP 190' da PM de Curitiba, cujo principal objetivo é acionar viaturas em situações de acidentes de trânsito, roubos, furtos, perturbação de sossego ou violência doméstica [Santos 2019]. O 'Alerta Cidadão' no RN, que permite o cadastro e acompanhamento de alertas de roubo, furto e perda de celulares [Lima 2018]. E o 'CITZs' cujas funcionalidades são o envio de alertas de violência e desordem em tempo real para as pessoas da região e também para o poder público, o recebimento de notificações em tempo real de casos ao redor do usuário e alertas sobre os casos que acontecem nos endereços de interesse cadastrados[Alex 2019].

De fato, como observado por [Baechler et al. 2017] em uma revisão de literatura a respeito de aplicações móveis que suportam investigadores em locais de crime, existe uma lacuna na literatura de ciências forenses sobre esse tipo de soluções. Os autores afirmaram ainda que, apesar da popularidade de pesquisas sobre desenvolvimento de aplicações móveis, pesquisas sobre aplicações móveis voltadas à investigação de locais de crime ainda estão em fases iniciais e, portanto, há ainda pouco conhecimento científico consolidado. Neste estudo, respostas de policiais a um questionário confirmaram a falta de aplicações móveis específicas para apoiar investigações em locais de crime. Alguns policiais relataram que usam aplicativos móveis de propósito geral (câmera fotográfica, notas 
de texto, aplicativos de comunicação, etc) para apoiar tarefas investigativas. Desta forma, observa-se que aplicativos para locais de crime têm grande potencial para apoiar equipes de investigadores em locais de crime.

\section{Conclusão}

A violência no Brasil é uma preocupação significativa nas vidas dos cidadãos e dos órgãos públicos, que trabalham diariamente para combater seu crescimento. Os crimes graves envolvendo homicídios afetam diretamente os fatores socioeconômicos da sociedade, deixando marcas profundas. Por esses motivos a segurança é um aspecto extremamente relevante para o sucesso de uma Cidade Inteligente, pois afeta diretamente a vida dos seus cidadãos em diversos sentidos.

Partindo dessa carência na segurança publica e as dificuldades relatadas pelos funcionários da DHPP, o ACC integra soluções de Cidades Inteligentes para auxiliar agentes de investigação em campo. Com validação de todas suas funcionalidades, pelos delegados da DHPP, o sistema está pronto para apoiar as atividade de investigação na cena do crime. Assim é possível substituir o cansativo e arcaico processo de cadastros de ocorrências por meio de papel e caneta por um método moderno e eficaz possibilitado um fácil cadastro e investigação futura.

\section{Referências}

Alex (2019). Citzs chegou! Disponível em:http://tinyurl.com/yytx4tjv. Acesso em Março 29, 2019.

Baechler, S., Gélinas, A., Tremblay, R., Lu, K., and Crispino, F. (2017). Smartphone and tablet applications for crime scene investigation: State of the art, typology, and assessment criteria. Journal of forensic sciences, 62(4):1043-1053.

Coelho, J., Cacho, N., Lopes, F., Loiola, E., Tayrony, T., Andrade, T., Mendonça, M., Oliveira, M., Estaregue, D., and Moura, B. (2016). Rota: A smart city platform to improve public safety. In New Advances in Information Systems and Technologies.

Lima, H. (2018). Aplicativo ajuda população na recuperação de celulares roubados. Disponível em:http: / / tinyurl . com/yypab juh. Acesso em Março 29, 2019.

Mingardi, G. and Figueiredo, I. (2005). A investigação de homicídios: construção de um modelo. Brasília: Ministério da Justiça.

Moraes, A. d. et al. (2014). Investigação criminal de homicídios. Brasília : Ministério da Justiça, Secretaria Nacional de Segurança Pública (SENASP), 2014. 124 p. - (Caderno temático de referência). Disponível em:http://tinyurl.com/ y 5 jgwen 4. Acesso em Março 29, 2019.

Santos, M. (2019). Policia militar lança versão do app 190 - emergência paraná para sistema ios e divulga balanço de uso pela população da grande curitiba. Disponível em:http: / / tinyurl. com/y38evca3. Acesso em Março 29, 2019.

Souza, E., Minayo, M., and Minayo, M. (2005). Violência, um problema para a saúde dos brasileiros: introdução. Impacto da violência na saúde dos brasileiros.

Tandel, S. and Jamadar, A. (2018). Impact of progressive web apps on web app development. International Journal of Innovative Research in Science, Engineering and Technology, 7(9):9439-9444. 